\title{
Efeito de diferentes tamanhos de clareiras, sobre o crescimento e a mortalidade de espécies arbóreas, em Moju-PA ${ }^{1}$
}

\author{
Fernando Cristóvam da Silva JARDIM², Dinilde Ribeiro SERRÃO3 ${ }^{3}$, Tangrienne Carvalho NEMER ${ }^{3}$
}

\section{RESUMO}

Crescimento e mortalidade de Sterculia pruriens, Vouacapoua americana, Jacaranda copaia, Protium paraensis, Newtonia suaveolens e Tabebuia serratifolia, considerando diferentes tamanhos de clareiras, foram avaliados em Moju - PA(207’30" e $2^{\circ} 12^{\prime} 06^{\prime \prime}$ de latitude Sul e 4846’57" e 4848'30" de longitude a Oeste de Greenwitch). Selecionou-se nove clareiras da exploração florestal, que foram agrupadas em pequenas $\left(200 \mathrm{~m}^{2} \leq\right.$ Área $\left.<400 \mathrm{~m}^{2-}\right)$ médias $\left(400 \mathrm{~m}^{2} \leq\right.$ Área $\left.<600 \mathrm{~m}^{2}\right)$ e grandes $\left(\geq 600 \mathrm{~m}^{2}\right)$. Em seu torno instalouse parcelas quadradas de cinco metros de lado, nas direçôes Norte, Sul, Leste e Oeste, onde foram plantados indivíduos da regeneração natural de espécies arbóreas. No centro de cada clareira foi instalada uma parcela de $5 \mathrm{~m}$ X $5 \mathrm{~m}$ como comparador. A média da mortalidade total foi de $46,9 \%$, não havendo diferenças entre as clareiras pequenas(41,05\%) e médias (43,86\%), mas estas diferiram das grandes $(54,96 \%)$. As clareiras pequenas são mais propícias para a maioria das espécies, exceto para J. copaia e $N$. suaveolens, cujas mortalidades foram menores nas clareiras médias. A mortalidade variou de $14,5 \%(\mathrm{~S}$. pruriens $)$ nas clareiras pequenas a $70,1 \%(V$. americana) em clareiras grandes, sendo que $S$. pruriens mostrou menor mortalidade em todos os tamanhos de clareiras. As espécies morreram mais em clareiras grandes. A mortalidade está entre os valores encontrados na literatura, permitindo concluir que não se pode classificar com precisão as espécies em grupos ecológicos somente com base na mortalidade ou sobrevivência. Em termos de crescimento, os resultados indicam que os melhores sítios para desenvolvimento das espécies são as clareiras médias, seguidos pelas clareiras grandes e pequenas. Em termos gerais, a média de crescimento em altura foi de $11,34 \mathrm{~cm}$ e de $0,11 \mathrm{~cm}$ em diâmetro de base, com valores maiores para J. copaia. Somente $V$. americana e $P$. paraenses não apresentaram diferenças significativas no crescimento em altura em relação aos diferentes tamanhos de clareiras. Os valores de crescimento e mortalidade das espécies apresentaram variaçôes em relação aos diferentes tamanhos de clareiras. J. copaia e $N$. suaveolens apresentaram melhor desempenho, tanto em termos de mortalidade como de crescimento em altura e diâmetro de base nas clareiras médias, todavia essa mortalidade foi elevada em comparação com S. pruriens.

PALAVRAS-CHAVE

Crescimento e mortalidade de plântulas, regeneração natural, clareiras artificiais, floresta tropical. \section{Effect of diferente gap sizes on the growth and mortality of arboreal species, in Moju-PA}

\begin{abstract}
Growth and mortality of Sterculia pruriens, Vouacapoua americana, Jacaranda copaia, Protium paraensis, Newtonia suaveolens and Tabebuia serratifolia regarding different gap sizes, had been evaluated in Moju - Pará - Brazil(2 $2^{\circ} 07^{\prime} 30^{\prime \prime S}$ and $2^{\circ} 12^{\prime} 06^{\prime \prime S}$ and

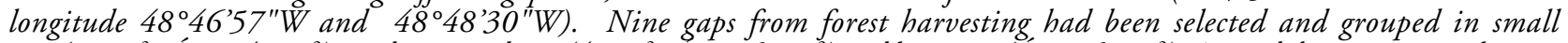
gaps $\left(200 m^{2} \leq\right.$ Area $\left.<400 m^{2}\right)$, medium-sized gaps $\left(400 m^{2} \leq\right.$ Area $\left.<600 m^{2}\right)$ and large gaps(Area $\left.\geq 600 m^{2}\right)$. Around the gaps square plots $5 m$ side had been installed, starting in the gap border to forest inside, according to North, South, East and West directions. In these plots seedlings from natural regeneration of tree species were planted. In the each gap center was installed a square plot of $5 m$ side as testimony, where the plantation was repeated. The total mortality average was 46.9\%, not showing significant differences between the small gaps (41,05\%) and medium-sized gaps (43,86\%) but these had differed from the large gaps (54,96\%). The small gaps environment was most favorable for the establishment of most species, except for $\mathrm{J}$. copaia and $\mathrm{N}$. suaveolens whose mortalities had been lesser in the medium-sized gaps, even so has not had significant differences in mortality between the different gap sizes. Mortality varied from 14,5\%(S. pruriens) in the small gaps to 70,1\%(V. americana) in large gaps, but S. pruriens showed lesser mortality in all sizes of gap. All species had shown greater mortality in large gaps, with remark for $\mathrm{V}$. americana $(70,1 \%)$, J. copaia $(69,1 \%)$ and $\mathrm{N}$. suaveolens $(58,7 \%)$. The mortality are between those finding in literature and for its variability they allow to conclude that if it cannot classify accurately the species in ecological groups only on mortality or survival basis. In terms of growth, however, the results are more conclusive, with evident indication of the medium-sized gaps as the best sites for development of the species, with average height growth of $15 \mathrm{~cm}$ and base diameter growth $0,16 \mathrm{~cm}$, followed for the large gaps and for small ones. In general terms, the height growth average was $11,34 \mathrm{~cm}$ and the base diameter ones was $0,11 \mathrm{~cm}$, with maximum values of $32,5 \mathrm{~cm}$ (height) and $0,24 \mathrm{~cm}$ (diameter of base) for J. copaia. P. paraensis and V. americana only had not presented significant differences in height growth regarding to different gap sizes, but the values had been a little bigger in the medium-sized gaps. The species growth and mortality, in two years of monitoring, had presented variations in relation to the different gap sizes. J. copaia and $\mathrm{N}$. suaveolens had presented performance better, as much in terms of mortality as of growth in height and diameter of base in the medium-sized gaps, however this mortality was high in comparison with S. pruriens. It must, therefore, be care when taking silvicultural decisions like as thinning, because it was verified differences even though between species considered of the same ecological group.
\end{abstract}

\section{KEY WORDS}

seedlings growth and mortality, natural regeneration, artificial gaps, tropical forest

${ }^{1}$ Estudo do projeto "Rendimento sustentável em floresta tropical manejada", financiado pelo CNPq e FUNTEC

${ }^{2}$ Engenheiro Florestal, Dr, Prof. Adjunto IV, Instituto de Ciências Agrárias/UFRA. ferrnando.jardim@ufra.edu.br

${ }^{3}$ Engenheira Florestal, MsC. Ciências Florestais UFRA. fjardim@nautilus.com.br 


\section{INTRODUÇÃo}

O manejo florestal tem, no conhecimento da autecologia das espécies arbóreas, o suporte para sua execução em bases sustentáveis. Para a aplicação de tratamentos silviculturais ou para planejar a intensidade de exploração, torna-se necessário conhecer as exigências das espécies em relação à radiação, fator que desencadeia a atividade metabólica dos vegetais.

Por outro lado, as florestas tropicais têm na ocorrência de clareiras naturais, a base para a renovação de sua composição florística. Nesses ambientes, geralmente, a vegetação é diferenciada pelas mudanças no ambiente físico. A mudança da quantidade de radiação que chega até o piso florestal éo primeiro fator observado nesses ambientes, acompanhado da temperatura, umidade e de fatores bióticos, os quais podem ser bruscamente modificados dependendo da intensidade do distúrbio provocado.

O tamanho da abertura no dossel florestal é um parâmetro que também deve ser levado em consideração por influenciar na composição florística, muitas vezes determinando a distribuição espacial das espécies. Existem espécies que possuem uma estratégia de desenvolvimento adaptada para locais onde ocorre um distúrbio, o qual permitirá a entrada da radiação até o piso florestal, ativando o banco de sementes ou de plântulas. É provável que, devido às diferentes quantidades de radiação que chegam ao piso florestal, Martins (1999) tenha encontrado menor similaridade entre a clareira menor e a maior, por representar dois extremos em termos de abertura de dossel e, portanto, de luminosidade. Assim, ao contrário da maior clareira, a menor apresentou apenas uma espécie pioneira, sendo dominada por espécies do sub-bosque.

As clareiras variam em relação ao tamanho, que por sua vez, influenciam as condiçōes microclimáticas dentro das mesmas; em relação à freqüência temporal e espacial de ocorrência, que afetam a probabilidade de um propágulo chegar a uma clareira de determinado tamanho. As condiçôes microclimáticas no centro da clareira, especialmente luz, temperatura e umidade, são influenciadas pela forma, orientação e tamanho da clareira, os quais determinam a duração diária de insolação direta (Costa \& Mantovani, 1992).

De acordo com Denslow (1980), a distribuição da freqüência de clareiras de diferentes tamanhos em floresta tropical úmida é influenciada pelo clima local, topografia, solo, rocha matriz e a composição florística e distribuição dos tamanhos das árvores. Quanto maior o tamanho da clareira, maiores são os valores extremos dos parâmetros microclimáticos e da influência da penumbra (Brown, 1993).

$\mathrm{Na}$ maioria das florestas tropicais, clareiras grandes, envolvendo a morte de várias árvores, são mais raras que clareiras pequenas, envolvendo só uma árvore ou um galho de árvore. A coexistência de muitas espécies de árvores de floresta tropical é devida, pelo menos parcialmente, ao fato de elas dividirem clareiras no dossel florestal. Portanto, a característica da distribuição de frequiência de classe de tamanho de clareiras de uma determinada floresta tropical deve influenciar os tipos e diversidade das espécies presentes (Denslow, 1980).

Existe dificuldade em definir a escala de tamanho e a forma das clareiras (Brokaw, 1982; Whitmore et al, 1993). As comunidades resultam, geralmente, de distúrbios em escala pequena e, raramente, em escala grande (Almeida, 1989; Costa \& Mantovani, 1992; Tabarelli, 1994; Martins, 1999), o que leva a suposição de que existem poucas espécies adaptadas a clareiras nas florestas, onde o ritmo de fechamento nas clareiras pequenas é mais intenso (Denslow \& Hartshorn, 1994).

O tamanho de uma clareira natural varia, dependendo do agente causal da mesma, podendo ser pequena por decorrer $\mathrm{da}$ queda de pequenos galhos ou até hectares de área (Hartshorn, 1980). De qualquer forma, a curva de distribuição das clareiras em classes de tamanho segue um padrão exponencial decrescente, com maior concentração nas menores classes de tamanho.

Numa área que sofreu exploração florestal seletiva, o tamanho das clareiras é muito próximo, pois são aberturas provocadas pelo corte de uma ou mais árvores, dependendo da distribuição espacial das mesmas e da disponibilidade de espécies que estão sendo exploradas. Nesse caso, a curva da distribuição de tamanhos, embora ainda no formato exponencial decrescente, é mais achatada, devido a uma maior quantidade de clareiras de tamanho médio e grande. Por essa razão, a exploração florestal beneficia um conjunto de espécies com maior demanda por radiação e, muitas vezes, pode dispensar a aplicação de tratamentos silviculturais para estimular a regeneração natural das espécies comerciais, na sua maioria espécies com maior grau de héliofilia (Souza \&Jardim, 1993)

Para Carvalho (1999), haverá sempre uma diferença na dinâmica da composição florística entre uma clareira grande e uma pequena, ou entre diferentes intensidades de exploração, pois são afetadas a germinação e a sobrevivência através da influência sobre fatores físicos dentro da mesma. A entrada da radiação para o solo aumenta com o tamanho da clareira e decresce com a altura do dossel (Orians, 1982), existindo um aumento acentuado no fluxo de luz da menor para a maior clareira (Barton et al., 1989).

O tamanho da clareira é correlacionado significativa e positivamente com a abertura e altura do dossel (Martins, 1999). Onde o dossel florestal é mais alto, a queda de árvores maiores provoca a formação de clareiras maiores. Almeida (1989) registrou uma grande abundância de clareiras de diversos tamanhos na região de Manaus - Brasil, cuja área foi influenciada tanto pelo número como pelo tamanho das árvores formadoras das clareiras.

O tamanho da clareira tem fundamental importância para sucessão da floresta, sendo responsável pela dinâmica das espécies e um importante fator na manutenção da alta diversidade das 
florestas tropicais (Hartshorn, 1989). Pequenas clareiras, como aquelas formadas pela queda de um galho, normalmente não promovem as condiçôes microclimáticas para o estabelecimento de espécies pioneiras. Nessas condições, as espécies de clímax normalmente preenchem a clareira pelo crescimento lateral dos galhos. Por outro lado, se a clareira é grande, ela é primeiramente colonizada pelas espécies pioneiras. Assim, o tamanho das clareiras deve ser levado em consideração quando se planeja a exploração florestal (Silva, 1989). Quando a espécie desejável é tolerante à sombra, a exploração madeireira deve ser cuidadosamente planejada, minimizando a formação de clareiras muito grandes, que favoreceriam o desenvolvimento de espécies heliófilas indesejáveis.

Existem estudos em diferentes tamanhos de clareiras para obtenção de informações sobre o desempenho comparativo de mudas de espécies florestais com objetivo de apoiar a silvicultura e o manejo de florestas tropicais (Turner, 1990; Jardim, 1995; Jennings et al., 1997; Mory, 2000; Sena et al., 2000; Vasconcelos et al., 2000). Para Carvalho (1997), a manipulação da floresta durante as operações de exploração florestal deve ser feita cuidadosamente para proporcionar condições semelhantes para toda a área explorada, chamando atenção ao fato de que existem espécies que requerem cuidados especiais durante as operações de exploração para sobreviver após a mesma. Alguns pontos, tais como grau de abertura do dossel, época de disseminação de sementes, mecanismos de dispersão, proximidade de árvores matrizes e condiçôes edafoclimáticas, entre outros, devem ser considerados durante os tratamentos silviculturais.

A intensidade de radiação solar que entra na floresta deve ser suficiente para favorecer as espécies desejáveis. Portanto, o nível de abertura realizado no momento de uma exploração, ou mesmo para realização de tratos silviculturais, deve ser bem planejado, haja vista que, em sítios muito perturbados, a resiliência é baixa, tomando muito tempo entre o aparecimento das espécies colonizadoras e o estabelecimento dos grupos ecológicos que apresentam espécies de importância silvicultural (Miranda, 1993). Todavia, as informaçôes acerca do comportamento das espécies florestais são escassas, havendo necessidade de muitos estudos para subsidiar a prescrição de tratamentos silviculturais adequados.

Este trabalho tem por objetivo gerar informaçôes a respeito do crescimento e da mortalidade de mudas de seis espécies arbóreas transplantadas da regeneração natural, levando em consideração o tamanho de clareiras formadas na exploração florestal seletiva, no município de Moju - Pará, como subsídios para a silvicultura nos planos de manejo florestal. Portanto, testouse a hipótese de que o tamanho das clareiras influencia no comportamento das espécies.

\section{MATERIAL E MÉTODOS}

\section{CARACTERIZAÇÃO DA ÁREA}

O experimento foi conduzido em uma área de 200 ha no campo experimental da EMBRAPA Amazônia Oriental, localizado no km 30 da Rodovia PA-150, no município de Moju$\mathrm{PA}$, situado entre as coordenadas geográficas de $2^{\circ} 08 \$$ e $2^{0} 12 \phi$ de Latitude sul e $48^{\circ} 47 \$$ e $48^{\circ} 484$ de Longitude Oeste de Greenwich, a qual sofreu exploração seletiva no período de Outubro de 1997.

A cobertura vegetal da região consiste de uma floresta tropical densa de terra-firme, constituída por árvores de grade porte, com altura do dossel situando-se numa faixa de 25-35 m.

O clima é Ami, de acordo com a classificação de Köppen, com temperatura média anual entre $25-27^{\circ} \mathrm{C}$ e precipitação anual entre $2000-3000 \mathrm{~mm}$, com distribuição irregular, ocorrendo pequeno período seco. A insolação mensal varia entre 148-275,8 horas, com os valores mais elevados ocorrendo no período de junho a dezembro e apresentando estreita relação com a precipitação.

O relevo é plano, com pequenos desnivelamentos com declive variando de $0-3 \%$. O solo predominante éo Latossolo Amarelo, com diferentes texturas, ocorrendo também Podzólicos Vermelhoamarelos, Glei Pouco Húmico e Plintossolos (Santos et al., 1985).

O experimento foi instalado em 1998 quando foram selecionadas nove clareiras, todas originadas da exploração florestal feita na área, com tamanho variando de $231 \mathrm{~m}^{2}$ a $748 \mathrm{~m}^{2}$. Cada clareira teve seu centro determinado através de processos topográficos (bússola e trenas). Em cada clareira foram instaladas parcelas quadradas de $5 \mathrm{~m}$ de lado na bordadura da clareira, a 20 m e $40 \mathrm{~m}$ para dentro da floresta, nas direçōes Norte, Sul, Leste e Oeste (Figura 1), onde foram plantados, aleatoriamente e em espaçamento de $1 \times 1 \mathrm{~m}, 36$ indivíduos por parcela de seis espécies arbóreas, variando de espécies heliófilas a espécies tolerantes, conforme sugerido por Jardim (1995). Como testemunha, no centro de cada clareira, foi também instalada uma parcela de $5 \times 5 \mathrm{~m}$, onde foi feito o plantio semelhante às demais parcelas.

As espécies utilizadas no plantio foram: Jacaranda copaia Aublet (parapará), Protium paraensis (Aublet) Maech (breu), Sterculia pruriens Ducke (achichá), Vouacapoua americana Aublet (acapu), Tabebuia serratifolia (Vahl) Nicholson (ipê amarelo) e Newtonia suaveolens (Miq) Benon (timborana).

Com exceção de Vouacapoua americana (acapu), todas as mudas foram plantadas livres dos cotilédones, tendo sido obtidas da regeneração natural, coletadas à mão livre e selecionadas de acordo com o vigor e tamanho. $\mathrm{O}$ total de plantas por clareira foi de 468, totalizando 4.412 mudas no experimento. 


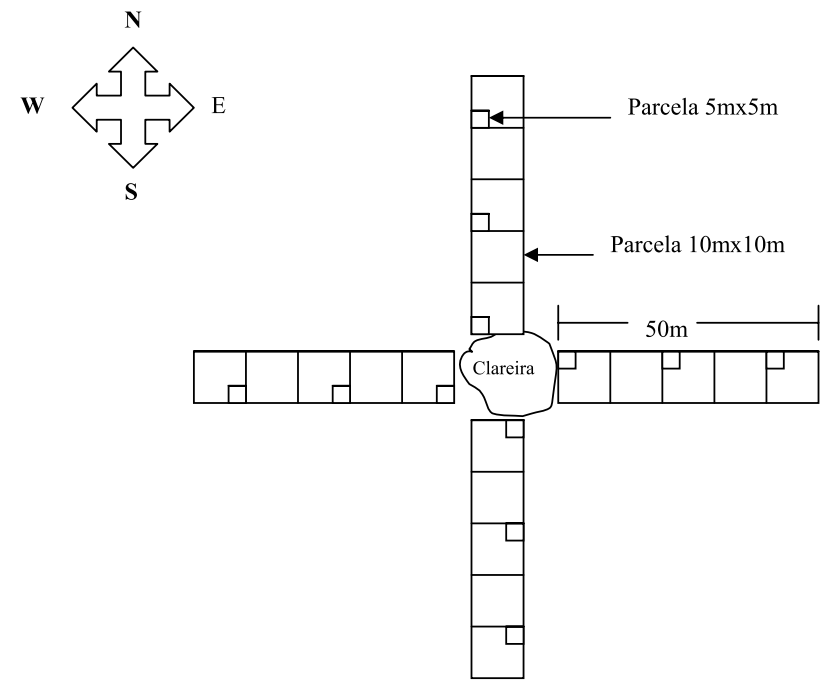

Figura 1 - Desenho esquemático da distribuição das parcelas amostrais de plantio, em relação às clareiras.

Imediatamente após o plantio (março de 1998) foi realizada uma medição da altura dos indivíduos para obtenção dos dados iniciais. Os dados deste estudo são referentes a um período de 24 meses após a instalação do experimento.

\section{COLETA E ANÁLISE DOS DADOS}

Para a realização deste trabalho, as nove clareiras utilizadas no estudo foram separadas convencionalmente em três classes de tamanho: Clareiras pequenas $\left(200 \mathrm{~m}^{2} \leq\right.$ Área $\left.<400 \mathrm{~m}^{2}\right)$; Clareiras médias $\left(401 \mathrm{~m}^{2} \leq\right.$ Área $\left.<600 \mathrm{~m}^{2}\right)$ e Clareiras grandes: (Área $\geq$ $\left.600 \mathrm{~m}^{2}\right)$.

As espécies utilizadas neste estudo foram Jacaranda copaia (Aubl) D. Don (parapará), Protium paraensis (Aubl) Maech (breu), Sterculia pruriens (Aubl) R. Schum (achichá), Vouacapoua americana Aubl(acapu), Tabebuia serratifolia (Vaal) Nicholson(ipê-amarelo) e Newtonia suaveolens (Miq) Benon(timborana). No momento do transplantio cada espécie tinha alturas médias de 9,6 cm para Jacaranda copaia, $9,9 \mathrm{~cm}$ para Protium paraensis, $11,1 \mathrm{~cm}$ para Sterculia pruriens, $23,6 \mathrm{~cm}$ para Vouacapoua americana, $6,8 \mathrm{~cm}$ para Tabebuia serratifolia e $6,7 \mathrm{~cm}$ para Newtonia suaveolens.

Com os resultados coletados em junho de 1998 e junho de 2000 foram realizadas as análises da mortalidade, para a qual foram contados todos os indivíduos mortos durante os dois anos de observação, e do crescimento em altura e diâmetro de base, calculados para todos os indivíduos vivos. O Incremento Médio Anual, em altura e diâmetro de base foi calculado através da expressão:

$I M A_{(\text {AlturatotalouDiâmetrodebase })}=\frac{\text { mediçãofinal }- \text { mediçãoinicial }}{2(\text { anos })}$
O delineamento consiste em um fatorial inteiramente casualizado, com a interação tamanho de clareira x espécie. A análise dos dados foi realizada através do programa SAS. Para o crescimento foi realizado teste de médias, através do teste Duncan, a um nível de 0,05 de probabilidade, quando as interações foram significativas. Para mortalidade foi calculada a frequêencia dentro de cada interação.

\section{RESULTADOS E DISCUSSÃO}

\section{MORTALIDADE}

Levando-se em consideração o tamanho das clareiras (Tabela 2.1), foi observado um aumento significativo na mortalidade com o aumento do tamanho das clareiras. Isso se deve, provavelmente, em virtude da maior modificação causada no ambiente físico nas clareiras grandes, o que reduziu as condiçôes para o estabelecimento de plântulas, uma vez que a distribuição espectral da radiação no interior da floresta é completamente diferente daquelas em abertura como as clareiras (Lee, 1987).

$\mathrm{O}$ ambiente de clareiras pequenas foi mais propício para o estabelecimento da maioria das espécies (Figura 2), exceto para Jacaranda copaia e Newtonia suaveolens, as quais obtiveram menor mortalidade em clareiras médias. Apesar de não haver diferença significativa para nenhuma das espécies isoladas, entre os diferentes tamanhos de clareiras, é observado, nessa fase de vida, que a maioria das espécies requer ambientes com radiação mais amena, como condição ideal de estabelecimento. Isso confirma

Tabela 1 - Média da mortalidade de seis espécies arbóreas em diferentes tamanhos de clareiras da exploração florestal seletiva, em Moju-Pará, em dois anos de observação.

\begin{tabular}{lll}
\hline Tamanho de clareira & Mortalidade (\%) & $\mathrm{Z}=$ ArcSen $\sqrt{\text { Mortalidade }}(\%)$ \\
\hline Pequena & 41,05 & $0,68744^{\mathrm{B}}$ \\
Média & 43,86 & $0,7259^{\mathrm{B}}$ \\
Grande & 54,96 & $0,86517^{\mathrm{A}}$ \\
\hline
\end{tabular}

as afirmaçōes de que em clareiras muito grandes, plântulas e mudas já estabelecidas podem crescer pouco ou mesmo morrer em virtude da alta carga de radiação (Whitmore, 1978).

Apesar de ser pequena a diferença ambiental, em decorrência dos tamanhos de clareiras utilizados neste estudo, quando comparados com a literatura (Costa \& Mantovanni, 1992; Vieira \& Higuchi, 1990), onde existe uma diferença maior entre os tamanhos de clareiras, é constatado que a mortalidade, independente da espécie, foi mais acentuada em clareiras grandes (Tabela 2.1).

Brown (1993) encontrou uma forte relação entre tamanhos de clareiras e as variáveis microclimáticas em floresta tropical na ilha de Bornéu (Malásia). Ele constatou que, em condição natural, a sobrevivência e o crescimento das plântulas, tanto de espécies 


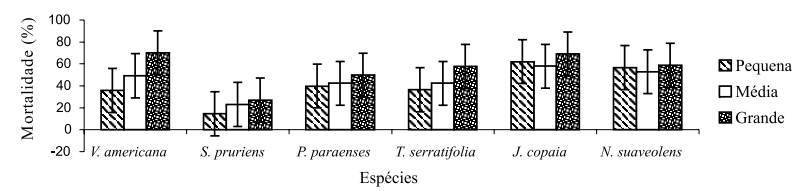

Figura 2 - Mortalidade de seis espécies arbóreas em diferentes tamanhos de clareiras da exploração florestal seletiva, em Moju-PA, em dois anos de observação.

pioneiras como espécies clímax, dependem muito dos fatores lumínicos das clareiras da floresta.

Jacaranda copaia, uma espécie tipicamente pioneira, mostrou alta mortalidade em todos os tamanhos de clareiras e, assim como Newtonia suaveolens, apresentou menor mortalidade em clareiras médias. A grande mortalidade de Jacaranda copaia pode ser característica dessa espécie, haja vista ter-se observado um grande número de indivíduos regenerando em ambientes que recebiam radiação direta. Um exemplo disse ocorre em estradas de arraste, aberturas provocadas pela queda de árvores da exploração florestal e em pátios de estocagem, porém neste estudo, foi considerado não só os indivíduos plantados no centro da clareira, mas também aqueles plantados no interior da mata.

Os resultados de Vieira \& Higuchi (1990) também foram compatíveis com este trabalho, pois concluíram que o tamanho das clareiras influenciou na mortalidade da regeneração natural em uma floresta tropical, ocorrendo as maiores taxas nas grandes clareiras. Estes resultados indicam que deve ser feito um bom planejamento do nível de abertura do dossel, evitando assim o favorecimento de espécies indesejadas, pois em ambientes onde ocorre uma abertura excessiva ocorre o ingresso de espécies ruderais, espécies de início de sucessão, levando um tempo maior, que pode chegar a 20 anos (Putz, 1984; Tabanez, 1995), para o surgimento de espécies arbóreas.

Das 4212 mudas plantadas, 46,9\% morreram em dois anos de observação. Turner (1990), ao realizar estudo em uma floresta tropical úmida na Malásia, inventariou 1287 plântulas menores que um metro de altura, em quatro clareiras naturais e dois sítios sob dossel fechado. Desse total, 251 (19,5\%) morreram durante um período de 16 meses de observação (14,6\%/ano). Ao comparar com esses valores é constatado que, neste estudo, a mortalidade foi elevada, principalmente, em Jacaranda copaia, e Newtonia suaveolens (Figura 2).

As diferentes taxas de mortalidade entre as espécies foram atribuídas mais às características da própria espécie, pois cada espécie tem um tipo de resposta a aberturas no dossel, embora a maioria tenha uma resposta positiva a ambientes com maior incidência de radiação solar. Jacaranda copaia e Sterculia pruriens apresentaram as maiores e menores taxas de mortalidade total, com médias de 63,0 \% e 21,5\%, respectivamente, e S. pruriens mostrou menor mortalidade em todos os tamanhos de clareiras.
Estudo na mesma área constatou que a taxa de regeneração natural de Sterculia pruriens foi maior em clareiras médias, seguidas das pequenas e grandes (Sena et al., 2000). Neste estudo essa espécie mostrou a melhor performance de estabelecimento no ambiente de clareiras pequenas (Figura 2), com mortalidade de $14,5 \%$. Entretanto, nas outras categorias de tamanhos de clareiras, a mortalidade não foi tão elevada quanto das outras espécies analisadas no período, verificando-se nessa espécie uma grande capacidade de adaptar-se em ambientes alterados.

Todas as espécies mostraram maior mortalidade nas clareiras grandes, com maiores valores para. Vouacapoua americana $(70,1$ $\%)$, Jacaranda copaia (69,1 \%) e Newtonia suaveolens (58,7 \%). Nas clareiras pequenas e médias, Jacaranda copaia e Newtonia suaveolens apresentaram as maiores mortalidades. A alta mortalidade dessas duas espécies em todos os tamanhos de clareiras pode ter ocorrido devido ao plantio das mesmas também no sub-bosque, além do centro das clareiras, uma vez que foi observada uma alta germinação de ambas as espécies em ambientes abertos da floresta, provocados pela exploração florestal, como por exemplo, em estradas de arraste e em clareiras da exploração.

A mortalidade das plântulas de Jacaranda copaia e Protium paraenses, dezoito meses após o plantio, já atingia valores de 58,2 $\%$ e $41,0 \%$, respectivamente (Vasconcelos et al., 2000). Neste trabalho, nas clareiras pequenas, médias e grandes, Jacaranda copaia apresentou uma mortalidade de 62,0 \%, 57,9 \% e 69, 1 $\%$ e Protium paraenses uma mortalidade de $39,7 \%, 42,3 \%$ e $49,8 \%$, respectivamente.

Esses resultados são compatíveis com os de Brokaw (1987) e Tabarelli (1994), quando estudaram clareiras de diferentes tamanhos e obtiveram resultados variados quanto ao comportamento de diferentes espécies, nesses ambientes.

Para Protium paraenses, foi constatado que, apesar de ter caráter tolerante à sombra, essa espécie pode se adaptar em clareiras, tendo taxa de mortalidade menor que espécies heliófilas como é o caso de Jacaranda copaia. Portanto, em ambientes de radiação solar direta, essa espécie mostrou um comportamento satisfatório. Lopes (1993) constatou que Protium apiculatum obteve melhor resultado em clareiras, apesar de ser considerada uma espécie tolerante, como Protium paraenses. Por outro lado, Eschweilera odora, uma espécie tipicamente tolerante, não reagiu a diferentes intensidades de desbaste (Mory \& Jardim, 2001a), constatando a grande diferença existente entre espécies pertencentes ao mesmo grupo ecológico.

Embora as clareiras tenham sido separadas em classes de tamanho, a magnitude dessas classes implicou no mesmo grau de alteração microclimática, motivo pelo qual Newtonia suaveolens e Jacaranda copaia apresentaram poucas diferenças em seu comportamento em relação aos tamanhos de clareiras. A forma e o tamanho de uma clareira são extremamente variáveis, podendo determinar que espécie possa colonizá-la com sucesso (Hartshorn, 
1980), pela interação com as características genéticas das espécies. Provavelmente, se houvesse maior diferença entre os tamanhos das clareiras, as respostas seriam mais características, uma vez que Jacaranda copaia precisa de radiação solar direta para realizar seu desenvolvimento.

Espécies que demandam aberturas para regenerar-se, como Jacaranda copaia e Sterculia pilosa (Caravalho, 1992), apresentaram um nível variado em relação à mortalidade, sendo esse comportamento decorrente, provavelmente, das características intrínsecas dessas espécies, ou até mesmo, do período sucessional a que pertencem, haja vista que espécies pertencentes ao mesmo grupo ecológico, podem fazer parte de diferentes fases sucessionais (Brokaw, 1987).

Tabebuia serratifolia, uma espécie heliófila, apresentou mortalidade mais próxima de Protium paraenses do que de Jacaranda copaia, que é uma espécie pertencente ao mesmo grupo ecológico (Figura 2). Portanto, observam-se espécies que apesar de pertencerem ao mesmo grupo ecológico, apresentam comportamento variado. Isso leva a crer que T. serratifolia é uma espécie que requer radiação direta para germinação. Entretanto, consegue estabelecer-se em ambiente com baixo nível de radiação, enquanto J. copaia demanda altos índices de radiação para a germinação de suas sementes e estabelecimento, sendo visível seu sucesso em local de exploração florestal (Veríssimo et al., 1989; Silva et al., 1995), da mesma forma como Goupia glabra, uma espécie heliófila, cujo surgimento é notado em clareiras onde ocorre uma alta quantidade de radiação até o piso florestal (Mory \& Jardim, 2001b).

A mortalidade de Sterculia pruriens pode ser considerada baixa quando comparada à mortalidade de Jacaranda copaia. Carvalho (1992) caracteriza Sterculia pilosa como uma espécie heliófila enquanto que Lopes (1993) classifica essa mesma espécie como uma espécie tolerante a sombra, portanto a mesma espécie, para dois autores, faz parte de grupos ecológicos diferentes. Sterculia pruriens, que é uma espécie pertencente ao mesmo gênero, possui mais características de espécie heliófila do que tolerante, podendo ser enquadrada em um grupo de espécies intermediárias, pois é conhecido que essa espécie precisa de radiação direta em alguma fase de sua vida, sendo notado seu bom desempenho em ambientes abertos pela exploração seletiva.

Ao comparar a mortalidade entre espécies em cada tamanho de clareiras (Figura 3) foi observado que esta foi variada, em todos os tamanhos de clareiras, apesar dessa diferença não ser estatisticamente significativa. As espécies Jacaranda copaia e Newtonia suaveolens apresentaram maior mortalidade em quase todos os tamanhos de clareiras, exceto nas clareiras grandes onde a mortalidade maior foi de Vouacapoua americana. Sterculia pruriens foi a espécie com menor mortalidade em todos os tamanhos de clareiras.
Protium paraenses, que é uma espécie considerada tolerante, apresentou a mesma mortalidade em clareiras médias (42,3\%) apresentada por Tabebuia serratifolia, uma espécie considerada heliófila, que só germina em ambientes de clareiras. É provável que a mortalidade de Vouacapona americana seja diminuída pela grande quantidade de reserva dos cotilédones de suas sementes que são uma ajuda no estabelecimento de plântulas (Whitmore, 1984). Além disso, clareiras geram uma variedade de nichos de regeneração, que atendem as necessidades de espécies distintas ecologicamente, e controlam a abundância de espécies tolerantes (Martins, 1999).

Os resultados deste estudo comprovam que as espécies apresentam uma variedade muito grande de respostas, mesmo sendo pertencentes ao mesmo grupo ecológico, o que indica que as mesmas requerem diferentes ambientes, sendo o tamanho de clareiras determinante na colonização da área, portanto tornandose necessário o acompanhamento das espécies nesses ambientes.

\section{CRESCIMENTO}

$\mathrm{O}$ incremento médio anual em altura para todas as espécies foi de $11,34 \mathrm{~cm}$ e em diâmetro foi de $0,11 \mathrm{~cm}$. Independentemente do tamanho da clareira, a espécie que apresentou maior crescimento foi Jacaranda copaia, com 32,5 $\mathrm{cm}$ em altura e 0,24 cm em diâmetro. Essa espécie é caracterizada por apresentar alto índice de regeneração e crescimento em ambientes abertos (Veríssimo et al., 1989). Campos (1998) indicaram essa espécie para reconstrução de florestas, por apresentar bom desenvolvimento a pleno sol, com um Incremento Médio Anual (IMA) em diâmetro de 1,6cm.ano ${ }^{-1}$ e em altura de $1,7 \mathrm{~m} . \mathrm{ano}^{-1}$, sem problemas de ataques de pragas ou doenças, sendo considerada uma espécie de fácil propagação. Espécies intolerantes à sombra crescem mais rápido que as tolerantes, principalmente quan do são espécies pioneiras como essa (Carvalho, 1997).

Ao comparar o crescimento em relação aos tamanhos de clareiras, independente de espécies (Figura 4), foi constatado que houve diferença entre os mesmos. Nas clareiras médias, tanto o crescimento em altura total $(15 \mathrm{~cm})$, quanto em diâmetro $(0,16$ $\mathrm{cm}$ ) foram maiores, seguidos pelas clareiras grandes com 10,85

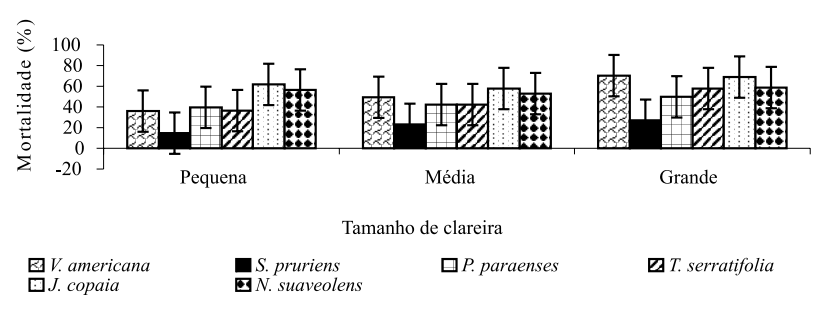

Figura 3 - Comparação da mortalidade entre seis espécies arbóreas, em diferentes tamanhos de clareiras em dois anos de observação em Moju-PA. 
$\mathrm{cm}$ de altura e $0,1 \mathrm{~cm}$ de diâmetro de base e clareiras pequenas com $8,16 \mathrm{~cm}$ (altura) e $0,2 \mathrm{~cm}$ (diâmetro de base). O ambiente de clareiras médias deve ser o que proporcionou melhor condição microclimática às espécies, uma vez que existe correlação entre tamanhos de clareiras e as variáveis microclimáticas em florestas tropicais. Em uma floresta tropical na ilha de Bornéu (Malásia) foi constatado que o crescimento das plântulas tanto de espécies pioneiras como espécies clímax, dependem muito dos fatores lumínicos das clareiras da floresta (Brown, 1993).

Ao comparar o crescimento das espécies em relação aos diferentes tamanhos de clareiras (Figura 5), foi observado que apenas Vouacapoua americana e Protium paraenses não apresentaram diferença no crescimento em altura em relação aos tamanhos de clareiras, apesar de ter havido um leve aumento no crescimento nas clareiras médias. Entretanto, o crescimento de

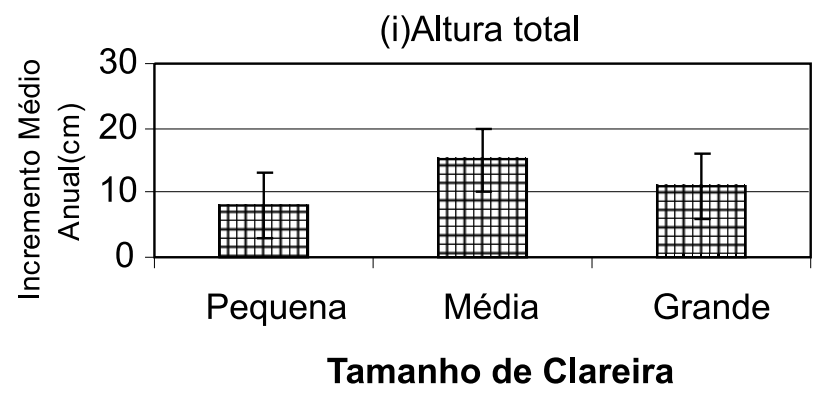

(ii)Diâmetro de Base

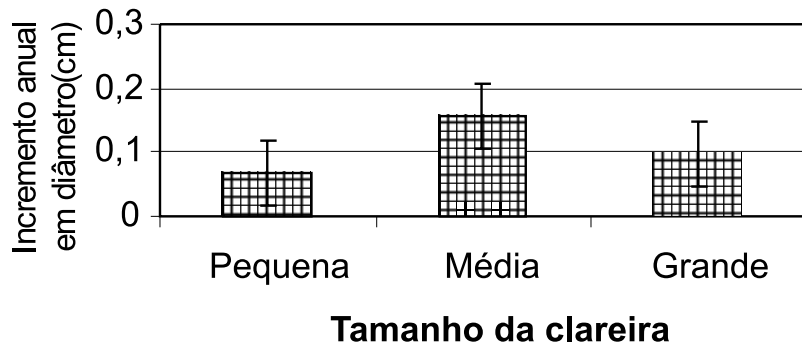

Figura 4 - Incremento Médio Anual em altura total (i) e diâmetro de base (ii) de seis espécies arbóreas, em relação a diferentes tamanhos de clareiras, em uma floresta explorada seletivamente, em Moju-PA. Médias seguidas pela mesma letra para cada parâmetro, não apresentam diferenças estatísticas entre si.

Vouacapoua americana foi mais acentuado que Protium paraenses. Isso se deve à sua própria característica, uma vez que Vouacapona americana é uma espécie que apresenta bom desenvolvimento em ambientes abertos.

Jacaranda copaia mostrou o maior crescimento em todos os tamanhos de clareiras, seguida de Sterculia pruriens e Vouacapona americana. O grande crescimento de J. copaia em todos os tamanhos de clareiras pode ser devido às clareiras em estudo serem todas grandes, quando comparadas a outros estudos (Costa \& Mantovani, 1992; Tabarelli, 1994; Vieira, 1995) e o microambiente formado por essas aberturas ser ideal para o crescimento dessa espécie. Por outro lado, mesmo considerando a grande mortalidade geral dessa espécie (Figura 2), os remanescentes podem ter se beneficiado do acréscimo de radiação no entorno do centro das clareiras, que pode ter atingido o dobro ou mais de $1 \%$ a $2 \%$ que chegam ao piso da floresta sob dossel fechado (Canham, 1989).

O aumento da área das clareiras e abertura do dossel resultou na redução na abundância relativa e aumento do diâmetro e área basal dos indivíduos (Martins, 1999). As clareiras menores foram mais ricas em espécies, mais abundantes e com menor área basal. Neste trabalho, todas as espécies mostraram crescimento significativo em diâmetro da base (Figura 5 ii), quando comparadas com os valores de $0,5 \mathrm{~mm} \cdot \mathrm{ano}^{-1}$ a $3,5 \mathrm{~mm}$.ano ${ }^{-1}$ das principais espécies do dossel na Ásia (Manokaran \& Kochummen, 1993), ou com o valor abaixo de $1 \mathrm{~mm} . \mathrm{ano}^{-1}$ para indivíduos com DAP $<10 \mathrm{~cm}$ de Dipteryx panamensis em La Selva, Costa Rica (Clark \& Clark, 1987), ou com valores menores que $5 \mathrm{~mm} /$ ano para espécies não pioneiras também em La Selva (Clark \& Clark, 1999). Os resultados deste estudo são lógicos, uma vez que a medida que os indivíduos crescem, alguns morrem, o que justifica a distribuição diamétrica decrescente, característica da maioria das espécies.

Espécies de valor comercial, geralmente, requerem aberturas no dossel para seu desenvolvimento. Uma comunidade mais valiosa vai possivelmente se desenvolver em clareiras próximas as árvores matrizes de espécies desejáveis (Carvalho, 1997). Algumas espécies comerciais, como Cordia goeldiana, regeneram melhor em solo descoberto, recebendo radiação solar direta, porém essa condição também pode favorecer o estabelecimento de algumas espécies pioneiras indesejáveis, como as do gênero Cecropia.

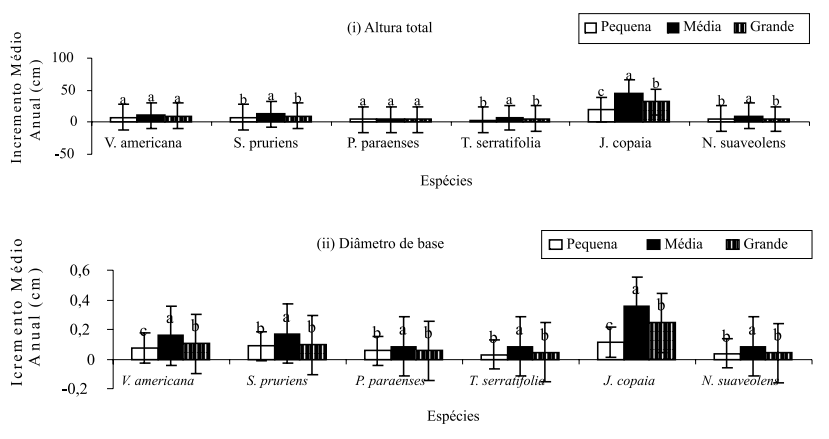

Figura 5 - Comparação do Incremento Médio Anual em altura total (i) e diâmetro de base (ii) das espécies em relação a diferentes tamanhos de clareiras em Moju - PA. Médias seguidas pela mesma letra, não apresentam diferenças estatísticas entre si 
Provavelmente, a pequena diferença entre os ambientes dos diferentes tamanhos das clareiras, seja responsável pela pequena diferença de crescimento das espécies com relação ao tamanho das clareiras (Figura 5). Todavia, na bacia do rio Cuieiras, ao Norte de Manaus, foi encontrado ampla variação de resultados desde crescimentos nulos $\left(0,0 \mathrm{~mm} \cdot \mathrm{ano}^{-1}\right)$ até $4,77 \mathrm{~mm} \cdot \mathrm{ano}^{-1}$, incluindo espécies comerciais como Eschweilera coriacea (DC. Mart. ex. Berg.) $\left(2,3 \mathrm{~mm} . \mathrm{ano}^{-1}\right)$, Protium altsonii Sandwith(1,8mm/ano), Scleronema micranthum Ducke(2,1 $\left.\mathrm{mm} . \mathrm{ano}^{-1}\right)$ e Virola calophylla Warb. $\left(2,4 \mathrm{~mm} . \mathrm{ano}^{-1}\right)$ (Silva et al., 2002).

Ao comparar o crescimento entre as espécies, dentro de cada tamanho de clareira (Figuras 2.5), foi constatado que existe diferença entre as espécies. Jacaranda copaia é a espécie que se destaca de todas as outras em todos os tamanhos de clareiras, por apresentar maior crescimento em altura e diâmetro da base. Vouacapoua americana e Sterculia pruriens, em todos os tamanhos de clareiras, não apresentaram diferença entre si, tanto em altura como em diâmetro da base, mostrando crescimento intermediário entre as espécies de menor crescimento como Protium paraenses e Tabebuia serratifolia e as de maior crescimento como Jacaranda copaia. Na região de Paragominas, PA, o maior crescimento diamétrico foi de Parkia gigantocarpa $\left(1,4 \mathrm{~mm} \cdot \mathrm{ano}^{-1}\right)$ e o menor foi de Lecythis lúrida (0,14 mm.ano ${ }^{-1}$ ) (Viddal et al., 2002).

Protium paraenses, Tabebuia serratifolia e Newtonia suaveolens não apresentaram diferenças entre si, exceto em clareiras pequenas, onde Tabebuia serratifolia apresentou menor crescimento que as demais em diâmetro de base. Espécies consideradas heliófilas como Jacaranda copaia e Tabebuia serratifolia, mostraram crescimento variado. Provavelmente, em decorrência de pertencerem a diferentes fases sucessionais, requerem radiação direta em diferentes fases de sua vida para realizar seu metabolismo, algumas tendo crescimento mais acelerado, como J. copia. Na Flona Tapajós, espécies intolerantes mostraram crescimento diamétrico de até $0,6 \mathrm{~cm} . \mathrm{ano}^{-1}$, incluindo J. Copaia $\left(0,4 \mathrm{~cm} \cdot \mathrm{ano}^{-1}\right)$ e espécies tolerantes mostraram crescimento de $0,23 \mathrm{~cm} \cdot \mathrm{ano}^{-1}$.

Essa variação de crescimento que ocorre entre espécies é comum, principalmente nas florestas tropicais onde ocorre uma grande variação entre microambientes, de acordo com o tamanho das clareiras, podendo haver variação até mesmo nos indivíduos de uma mesma espécie. Na bacia do rio Cuieiras, ao Norte de Manaus-AM, o incremento anual em diâmetro foi extremamente variado entre os indivíduos das espécies estudadas, com coeficiente de variação de 38\% (Goupia glabra Aubl.) a 431\%(Hevea guianensis Aubl.)(Silva et al., 2002).

Carvalho (1997) chama atenção ao fato de que o padrão de crescimento em diâmetro de certas espécies, pode ser semelhante em floresta virgem e em floresta explorada, enquanto que de outras pode ser completamente diferente. Na região de Manaus, por exemplo, o crescimento de espécies comerciais (listadas) variou de $0,17 \mathrm{~cm} \cdot \mathrm{cno}^{-1}$, em floresta não perturbada, até $0,30 \mathrm{~cm} /$ ano em floresta sob três níveis de exploração seletiva (Higuchi et al., 1997).

Favrin \& Kageyama (1989), ao avaliarem o estabelecimento de plântulas de duas espécies florestais, dentre elas Tabebuia avellanedae, constataram que, aos três meses de observação, ambas as espécies apresentaram uma tendência de maior número de plântulas estabelecidas no tratamento onde a luminosidade foi média. Para esses autores, tais resultados preliminares reforçam a hipótese de que as espécies secundárias tardias são mais tolerantes à sombra no seu estágio inicial de desenvolvimento. Portanto, apesar de Tabebuia serratifolia pertencer ao grupo de espécies heliófilas, o nível de radiação recebido no ambiente de clareira, não foi ideal para o seu desenvolvimento, ou então, o ritmo de crescimento dessa espécie é menos acelerado que de outras espécies heliófilas. Essa diferença pode ser notada na densidade da sua própria madeira, a qual é mais densa que de Jacaranda copaia.

Pelo que se observa, é comum acontecer diferenças de comportamento entre as espécies. Jacaranda copaia tem características de crescimento rápido, entretanto existem espécies que possuem maior capacidade de crescimento quando se encontram em ambientes propícios para melhor desenvolver seu metabolismo, como é o caso de Trema micrantha que cresce muito rápido atingindo até sete metros de altura em um ano, em clareiras maiores que $376 \mathrm{~m}^{2}$ (Brokaw, 1987).

Deve-se atentar ao fato de que o conceito de clareiras proposto engloba toda a área de influência da clareira, não sendo avaliados

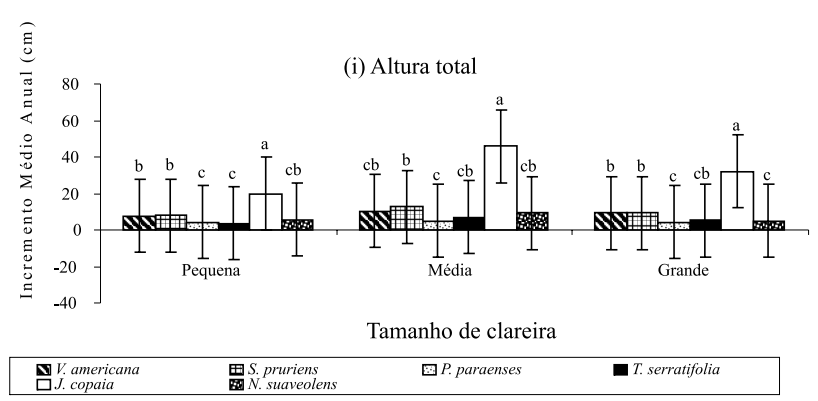

(ii) Diâmetro de base

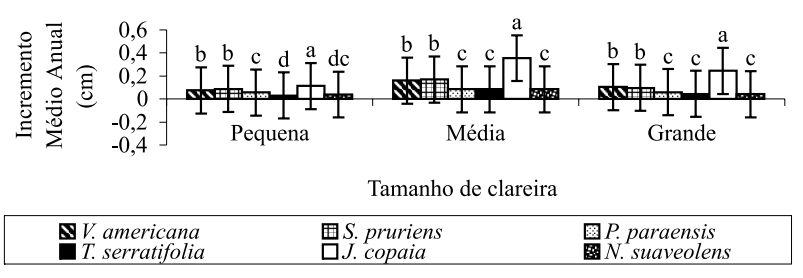

Figura 6 - Comparação, entre espécies, do Incremento Médio Anual em altura total (i) e diâmetro de base (ii), em diferentes tamanhos de clareiras em uma floresta explorada seletivamente em Moju-PA. Médias seguidas pela mesma letra, entre espécies, para o mesmo tamanho de clareira, não apresentam diferença significativa entre si. 
apenas os indivíduos plantados no centro da mesma. Existe comportamento variado de plântulas, dependendo de sua localização e se esse ambiente é favorável para seu desenvolvimento. Almeida (1989), ao realizar estudo em clareiras naturais de diferentes tamanhos em uma floresta tropical, observou que indivíduos de algumas famílias, localizados na margem das clareiras, tiveram maior crescimento em diâmetro, do que aqueles localizados no interior da mata, podendo-se atribuir isso a possíveis mudanças microclimáticas no ambiente das clareiras. Talvez existam nesses locais, além de uma quantidade de radiação adequada para seu desenvolvimento, diferentes quantidades de nutrientes, fornecidos pelo maior acúmulo de liteira.

Espécies tolerantes são as que apresentam esse tipo de comportamento, encontrando em pequenas clareiras ou margens de grandes clareiras ambiente ideal para seu estabelecimento. Protium paraenses, sendo uma espécie que possui caráter tolerante, mostrou menor crescimento em altura entre as espécies estudadas (Figuras 2.4i a 2.5ii). Provavelmente esse comportamento seja típico da mesma, haja vista esse grupo de espécies não ter estratégia de crescimento rápido na qual a radiação direta seja sua única possibilidade de sobrevivência. Esse grupo ecológico demonstra uma grande elasticidade ambiental, tendo capacidade de estabelecimento numa grande amplitude de ambientes, completando seu ciclo de vida em ambientes sombreados. Clareiras grandes provavelmente não devem ser seu ambiente ideal de estabelecimento, podendo afetar seu aparelho fotossintético, diminuindo ou até mesmo impedindo a realização de fotossíntese ou pela maior competição com espécies helófilas mais agressivas. Portanto, não só o ambiente mas as características intrínsecas das plantas são importantes para obtenção de sucesso no seu estabelecimento.

A variação de respostas que existe entre as espécies, nos diferentes tamanhos de clareiras (Figura 6), chama atenção ao fato de que, para a aplicação de tratamentos silviculturais em florestas tropicais, deve-se saber que nível de desbaste deve ser aplicado para conduzir o povoamento. Como pôde ser observado Jacaranda copaia é uma espécie que demanda um ambiente de radiação bastante definido. Espécies pioneiras aumentam sua abundância com o aumento da área de clareiras (Almeida, 1989). Todavia, espécies comerciais como Ocotea ociphylla, Licaria pachicarpa e Eschweilera coriacea não foram grandemente afetadas na sua densidade pela exploração florestal e somente Goupia glabra respondeu positivamente à abertura do dossel pela exploração seletiva(Lima et al., 2002)

Os resultados de Barton (1984), assim como de Almeida (1989), são coerentes com estes resultados. Barton (1984) mostrou que o estabelecimento de espécies pioneiras foi mais eficiente em clareiras grandes que em clareiras pequenas. Todavia, neste estudo, as clareiras que foram classificadas como médias são as grandes no estudo de Barton (1984). As espécies avaliadas neste estudo apresentaram poucas variaçōes entre os diferentes tamanhos de clareiras. É comum espécies pioneiras apresentarem maior densidade em clareiras grandes e espécies tolerantes à sombra não definirem precisamente seu ambiente. Martins (1999) destacou o papel das espécies tolerantes à sombra, que representaram entre $74 \%$ e $80 \%$ do total de espécies amostradas e entre $73 \%$ e $78 \%$ do total de indivíduos, concluindo que, como a maioria das clareiras era pequena, com área inferior a 200 $\mathrm{m}^{2}$, houve baixa disponibilidade de sítios para estabelecimento de espécies pioneiras.

Para definir que níveis de desbaste devem ser aplicados para conduzir espécies com comportamento tolerante à sombra ou intermediário, devem ser realizados um grande volume de trabalhos, em diferentes níveis de desbaste, por um período de tempo que permita a suficiência de informaçōes necessárias para a aplicação de técnicas silviculturais adequadas, haja vista que com o passar do tempo haverá o fechamento das clareiras, diminuindo o nível de radiação naquele ambiente.

\section{CONCLUSÕES}

Os valores de crescimento e de mortalidade das espécies, em dois anos de observação, apresentaram variações quanto ao comportamento em diferentes tamanhos de clareiras. Em virtude desse comportamento conclui-se que:

Sterculia pruriens apresentou baixa mortalidade em todos os tamanhos de clareiras, entretanto seu plantio deve ser feito em clareiras médias porque aí seu crescimento é maior;

Jacaranda copaia é uma espécie tipicamente heliófila, logo o seu plantio deve ser feito em ambientes de clareiras médias;

Deve-se ter cuidado no momento da tomada de decisões para aplicação de tratamentos silviculturais, como, por exemplo, níveis de desbaste, haja vista ter-se notado diferenças até entre espécies pertencentes ao mesmo grupo ecológico;

As espécies Jacaranda copaia e Newtonia suaveolens tiveram melhor desempenho tanto em mortalidade, quanto em crescimento em clareiras médias, apesar disso foi constatado que mesmo nesse tamanho de clareira a mortalidade foi elevada, por esse motivo devem ser realizados estudos para saber se a alta taxa de mortalidade observada é comum para essas espécies nesses ambientes;

A taxa média da mortalidade foi elevada pelos valores obtidos pelas espécies Jacaranda copaia, Vouacapoua americana e Newtonia suaveolens.

\section{BIBLIOGRAFIA CITADA}

Almeida, S.S. 1998. Clareiras naturais na Amazônia Central: abundância, distribuição, estrutura e aspectos da colonização vegetal. Dissertação de Mestrado. Instituto Nacional de Pesquisas da Amazônia/Universidade Federal do Amazonas. 1989. Manaus, Amazonas. 125 pp. 
Barton, A.M. 1984. Neo-tropical pioneer and shade-tolerant tree species: do they partition tree fall gaps. Tropical Ecology, 25: 196-202.

Barton, A.M.; Fetcher, N.; Redhead, S. 1989. The relationship between tree fall gap size and light flux in a Neo-tropical Rain Forest in Costa Rica. Journal of Tropical Ecology, 5: 437-439.

Bazzaz, F.A. 1991. Regeneration of tropical forests: Physiological responses of pioneer and secondary species. In: A. Gomes-Pompa, T.C. Whitmore and M. Hadley(eds). Rain forest regeneration and management. Paris: UNESCO. p. 91-118.

Brokaw, N.V.L. 1987. Gap-phase regeneration of three pioneer species in a tropical forest. Journal of Ecology, 75: 9-19.

Brokaw, N.V.L. 1982. Tree fall: frequency, timing, and consequences. In: The ecology of a tropical rain forest. Seasonal rhythms and long term changes. Washington DC: Smithsonian Institute. p. 101102.

Brown, N.D. 1993. The implication of climatic and gap microclimate seedlings growth conditions in a Bornean lowland rain forest. Journal of Tropical Ecology, 9: 133-168.

Campos, M.A.M. 1998. Seleção de espécies para sistemas agroflorestais com base no seu desempenho em plantios experimentais homogêneos e no potencial de uso múltiplo. In: Congresso Brasileiro em Sistemas Agroflorestais: no Contexto da Qualidade Ambiental e Competitividade, 2. Belém. Anais. Belém: EMBRAPA/CPATU. p. 20-22.

Canham, C.D. 1989. Different responses to gaps among shadetolerant tree species. Ecology, 70(3): 548-550.

Carvalho, J.O. 1992. Structure and dynamics of a logged over brasilian Amazonian rain forest. 1992. Doctor's Thesis. University of Oxford, Oxford. 215 pp.

Carvalho, J.O.P. de. 1997. Dinâmica de florestas tropicais e sua implicação para o manejo florestal sustentável. In: Curso de manejo florestal sustentável. 1997, Curitiba. Curitiba: EMBRAPA/CNPF. 253p. (Documentos, 34).

Carvalho, J.O.P. 1999. Dinâmica de florestas naturais e sua implicação para o manejo florestal. In: SIMPÓSIO SILVICULTURAL NA AMAZÔNIA ORIENTAL: Contribuiçōes do projeto EMBRAPA/DFID, Belém. Resumos expandidos. Belém: Embrapa. CPATU: DFID. p. 174-179.

Clark, A.; Clark, B. 1987. Análisis de la regeneración del dossel em bosque muy húmedo tropical: aspectos teóricos y práticos. Rev. Biol. Trop, 35(supl.1): 41-54.

Clark, D.A.; Clark, D.B. 1999. Assessing the growth of tropical rain forest trees: issues for forest modelling and management. Ecological Applications, 3(9): 981-997.

Costa, M.P.; Mantovani, W. 1992. Composição e estrutura de clareiras em mata mesófila na bacia de São Paulo, SP. In: Anais do $2^{\circ}$ Congresso Nacional sobre Essências Nativas. Revista Instituto Florestal, 4: 178-183.

Denslow, J.S. 1980. Gap partitioning among tropical rain-forest trees. Biotropica, 12:47-55.

Denslow, J.S.; Hartshorn, G.S. 1994. Tree-fall gap environments and forest dynamic process. In: McDade, L.; Bawa, K.S.; Hespenheide, H.A.; Hartshorn, G.S.(eds). La Selva-Ecology and Natural History of a Neotropical Rain Forest. The University of Chicago Press, Chicago. p. 120-127.

Favrin, L.J.B.; Kageyama, P.Y. Estabelecimento de plântulas de paineira (Chorisia speciosa st. Hil.) e ipê roxo (Tabebuia avellanedae, Lorentz) em condições de mata. In: SIMPÓSIO BRASILEIRO SOBRE TECNOLOGIA DE SEMENTES FLORESTAIS, 2. 1989, Atibaia. Anais. São Paulo: s.n. , 1989. p. 281.

Hartshorn,G.S. 1978. Neotropical forest dynamics. Biotropica, 12:3032.

Hartshorn, G.S. 1989. Gap-phase dynamics and tropical tree species richness. In: Holm-Nielsen, L.B. Nielsen I.C.; Basev, H. (eds). Tropical forests botanical dynamics, speciation and diversity. New York; Academic Press, New York. p 65-75.

Higuchi, N.; Santos, J.; Ribeiro, R.J.; Freitas, J.V.; Vieira, G.; Coic, A.; Minette, L.J. 1997. Crescimento e incremento de uma floresta amazônica de terra firme manejada experimentalmente. In: Higuchi, N.; Ferraz, J.B.S.; Antony, L.; Luizão, F.; Luizão, R.; Biot, I.; Hunter, I.; Proctor, J.; Ross, S.(eds). Bionte: Biomassa e nutrientes. Manaus: INPA/ DFID. p. 87-132.

Jardim, F.C.S. 1995. Comportamento da regeneração natural de espécies arbóreas em diferentes intensidades de desbaste por anelamento, na regiāo de Manaus - Am. Tese de Doutorado. Universidade Federal de Viçosa, Viçosa. 169pp.

Jennings, S.B. The response of tree seedlings to canopy disturbance in an Amazonian rain forest. 1997 . Doctors' Thesis. University of Oxford, Oxford. 195pp.

Lee, D.W. 1987. The spectral distribution of radiation in two neotropical rainforests. Biotropica, 19: 161-166.

Lima, A.P.; Lima, O.P.; Magnusson,W.E.; Higuchi, N.; Reis, F.Q. 2002. Regeneration of five commercial-valuable tree species after experimental logging in an Amazonian forest. Revista Árvore, 26(5):567-571.

Lopes, J.C.A. 1993. Demografia e flutuaçôes temporais da regeneração natural após uma exploração florestal: Flona do Tapajós-Pará. Dissertação de Mestrado. Universidade de São Paulo, Piracicaba. 133pp.

Martins, S.V. 1999. Aspectos da dinâmica de clareiras em uma floresta estacional semidecidual no município de Campinas, SP. Tese de Doutorado. Universidade de São Paulo, São Pauloz. 215pp.

Miranda, E.M. 1993. Efectos del aprovechamento de um bosque humedo tropical sobre el microambiente y su influencia em la regeneration de sitios perturbados. Dissertação de Mestrado Universidad de Costa Rica, Turrialba. 164pp.

Mory, A.M.; Jardim, F.C.S. 2001a. Comportamento de Eschweilera odora (Poepp.)Miers. (Matamatá-amarelo) em diferentes níveis de desbaste por anelamento. Revista de Ciências Agrárias, 36: 2953.

Mory, A.M.; Jardim, F.C.S. 2001b. Comportamento de Goupia glabra Aubl. (Cupiúba) em diferentes níveis de desbaste por anelamento em florestas naturais. Revista de Ciências Agrárias, 36: 55-66.

Orians, G.H. 1982. The influence of tree-fall in Tropical Forest on tree species richness. Tropical Ecology. 23(2): 255-279. 
Putz, F.E. 1984. The natural history of lianas on Barro Colorado Island, Panama. Biotropica, 19: 334-341.

Sena, J.R.C.; Jardim,F.C.S.; Jesus, R.T.; Serrão, D.R. 2000. Dinâmica da regeneração natural de Sterculia pruriens (Aubl) Schum. Sterculiaceae (axixá), em clareiras e áreas de influência das mesmas numa floresta explorada seletivamente, em Moju-PA. In: Seminário de iniciação científica da fcap, x e seminário de iniciação científica da embrapa amazônia oriental, IV, Belém. Resumos. Belém, 2000. Unidade de Apoio à Pesquisa e a PósGraduação, p. 340-343.

Silva, J.N.M. The behaviour of the tropical rain forest of the Brazilian amazon after logging. 1989, 302p. (Doctor Thesis) - University of Oxford, Oxford, 1989.

Silva, J.N.M; Carvalho, Lopes, J.C.; Almeida, B.F.; Costa, D.H.M.; Oliveira, L.C.; Vanclay, J.K. \& Skovsgaard, J.P. 1995. Growth and yield of a tropical rain forest in the Brazilian Amazon 13 years after logging. Forest ecology and management, 71: 267-274.

Silva, R.P.; Santos, J.; Tribuzy, E.S.; Chambers, J.Q.; Nakamura, S.; Higuchi, N. 2002. Diameter increment and growth patterns for individual tree growing in Central Amazon, Brazil. Forest Ecology and Management, 166: 295-301.

Tabanez, A.A.J. Ecologia e manejo de eco unidades em um fragmento florestal na região de Piracicaba, SP. 1995. Dissertação de Mestrado. Universidade de São Paulo, São Paulo. 85pp.

Tabarelle, M. Clareiras naturais e a dinâmica sucessional de um trecho de floresta na Serra da Cantareira, SP. 1994. Dissertação de Mestrado. Universidade de São Paulo, São Paulo. 132pp.

Turner, I.M. 1990. Tree seedlings growth and survival in a Malaysian Rain Forest. Biotropica, 22(2): 145-154.
Vasconcelos; L.M.R.; JESUS, R.T.; Serrão, D.R.; Vasconcelos, P.C.S.; Jardim, F.C.S. 2000. Mortalidade de mudas de Jacaranda copaia Aubl e Protium Paraenses Engl., em relação a diferentes tamanhos de clareiras e direções em floresta primária no município de Moju-PA. In: Seminário de Iniciação Científica da FCAP, X e Seminário de Iniciação Científica da Embrapa Amazônia Oriental, IV. 2000, Belém. Resumos. Belém: FCAP. Unidade de Apoio à Pesquisa e a Pós-Graduação, 173-176.

Veríssimo, A. et al. 1989. Impactos sociais, econômicos e ecológicos da exploração seletiva de madeiras numa região de fronteira na Amazônia Oriental: O caso de Tailândia. Pará Desenvolvimento, 25: $95-116$

Vidal, E.; Viana,V.M.; Batista, J.L.F. 2002. Crescimento de floresta tropical três anos após colheita de madeira com e sem manejo florestal na Amazônia Oriental. Scientia Forestalis, 61: 133-143.

Vieira, G.; Higuchi, N. 1990. Efeito do tamanho de clareira na regeneração natural em floresta mecanicamente explorada na Amazônia brasileira. In: Congresso Florestal Brasileiro, $6^{\circ}$. Campos do Jordão. Anais. São Paulo: s.n., 3: 666-672.

Vieira, G. 1995. Dynamics of the remaining plant population in gaps after logging in the Amazon. Conference on Forestry and Forest Products Research. p. 54-67.

Whitmore, T.C. 1978. Gaps in the forest canopy. In: TOMLINSON, Zimmerman. Tropical trees as living systems. Cambridge Univ. Press, London. p. 639-655.

Whitmore, T.C. et al. 1993. Use of hemispherical photographs in forest ecology: measurement of gap size and radiation totals in a Bornean tropical rain forest. Journal of Tropical Ecology, 9:131151.

Recebido em 06/10/2006

Aceito em 07/02/2007 
